\title{
Ultra-Sensitive Detection and Inhibition of the Metastasis of Breast Cancer Cells to Adjacent Lymph Nodes and Distant Organs by Using Long Persistent Luminescence Nanoparticles
}

Hanghang Liu, Feng Ren, Xingguo Zhou, Changqiu Ma, Tingting Wang, Hao Zhang, Qiao Sun*, Zhen Li*

Center for Molecular Imaging and Nuclear Medicine, State Key Laboratory of Radiation Medicine and Protection, School for Radiological and Interdisciplinary Sciences (RAD-X), Soochow University, Collaborative Innovation Center of Radiation Medicine of Jiangsu Higher Education Institutions, Suzhou 215123, P. R. China Email:sunqiao@suda.edu.cn zhenli@suda.edu.cn 


\section{Table of Contents}

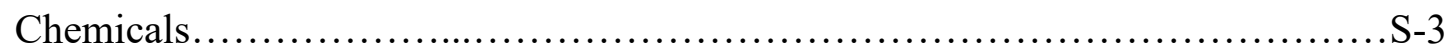

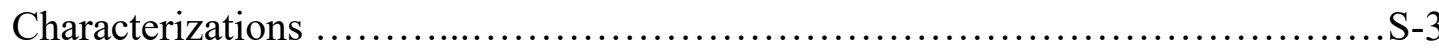

Preparation of $\mathrm{Cr}^{3+}$-doped, $\mathrm{Nd}^{3+}$-doped and $\mathrm{Cr}^{3+} / \mathrm{Nd}^{3+}$-codoped B-ZGC NPs........S-4

In vivo cytotoxicity assay and cell labeling ................................

Animal model and Histopathology examination................................. -5

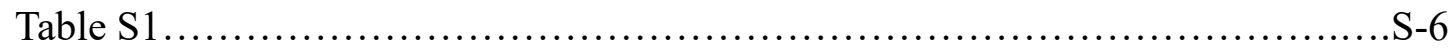

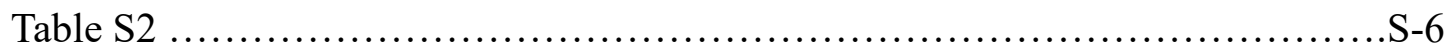

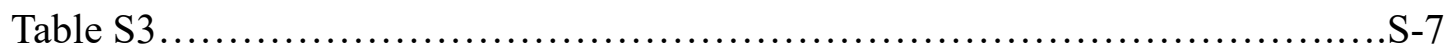

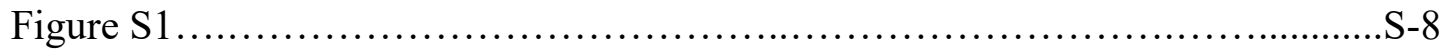

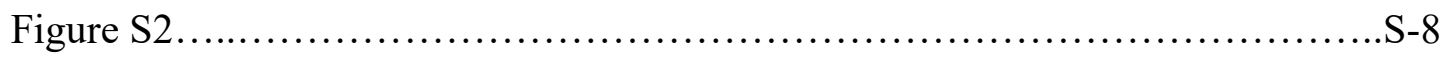

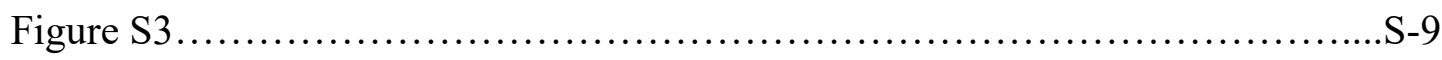

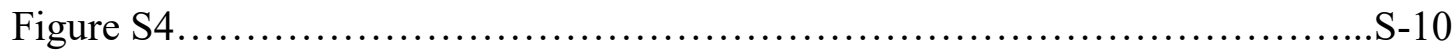

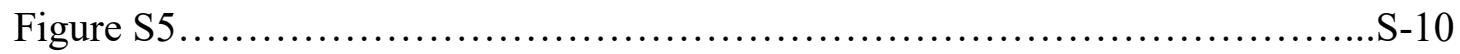

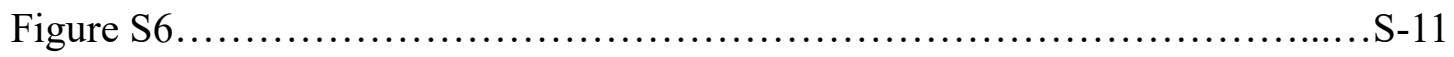

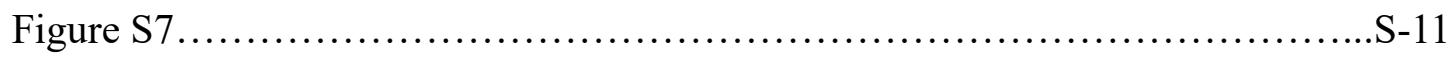

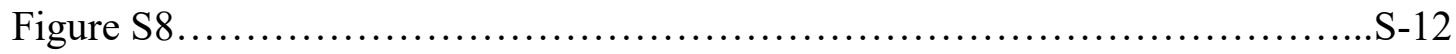

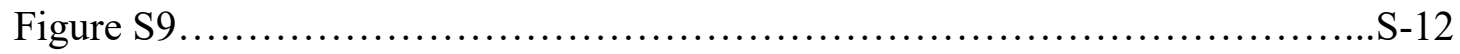

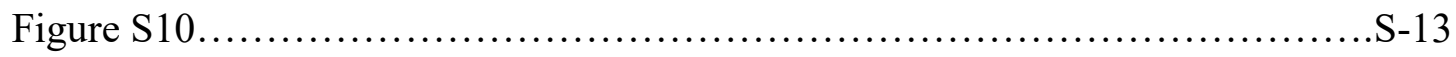

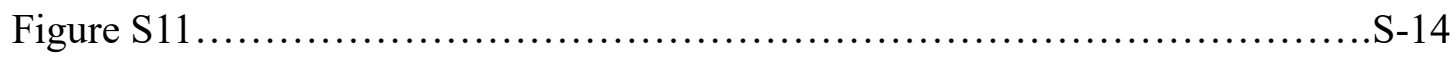

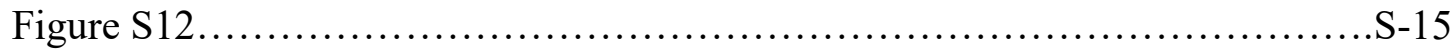


Chemicals. Neodymium nitrate hexahydrate $\left[\mathrm{Nd}\left(\mathrm{NO}_{3}\right)_{3} \cdot 6 \mathrm{H}_{2} \mathrm{O}, 99.9 \%\right]$ was purchased from Aladdin Co. Ltd. Zinc nitrate hexahydrate $\left[\mathrm{Zn}\left(\mathrm{NO}_{3}\right)_{2} \cdot 6 \mathrm{H}_{2} \mathrm{O}, 98 \%\right]$, gallium nitrate hydrate $\left[\mathrm{Ga}\left(\mathrm{NO}_{3}\right)_{3} \cdot x \mathrm{H}_{2} \mathrm{O}, 99.9 \%\right]$, chromium nitrate nonahydrate $\left[\mathrm{Cr}\left(\mathrm{NO}_{3}\right)_{3} \cdot 9 \mathrm{H}_{2} \mathrm{O}, 99 \%\right]$, sodium hydrogen carbonate, hydrochloric acid (analytical grade), isopropyl alcohol, N,N-dimethyllformamide were purchased from Sinopharm Chemical Reagent Co. Ltd. (Shanghai, China). 1, 2-distearoyl-sn-glycero-3phosphoethanolamine-polyethylene-glycol -carboxylic acid (DSPE-PEG-COOH, molecular weight, $\mathrm{M}_{\mathrm{W}}=5000$ ) was purchased from ToYongBio (Shanghai, China). (S)4,5-dihydro-2-(6-hydroxy-2-benzothiazolyl)-4-thiazolecarboxylic acid sodium salt was purchased from Shanghai Yeasen Biotechnology Co. Ltd. (Shanghai, China).

Characterizations. The morphology of the nanoparticles was characterized with a FEI Tecnai G20 transmission electron microscope (TEM) operating at an acceleration voltage of $200 \mathrm{kV}$. The high angle annular dark field-scanning TEM (HAADF-STEM) images were captured with a JEOLJEM-2010F. The hydrodynamic size and zeta potential were measured at $25^{\circ} \mathrm{C}$ using a Zetasizer Nano ZS90 dynamic light scattering instrument (Malvern). The excitation and emission spectra, as well as the decay curves of A-ZGC and A-ZGCN NPs were measured on a FLS980 spectrometer (Edinburgh Instruments, UK). The absolute photoluminescence quantum yields were measured as described elsewhere. ${ }^{1}$ The long lasting persistent luminescence decay curves and images were recorded on an IVIS Lumina II imaging system equipped with a CCD camera (PerkinElmer, America). Thermoluminescence (TL) spectra were collected using a FJ427A1 thermoluminescent dosimeter (CNNC Beijing Nuclear Instrument 
Factory). The NIR-I in vivo imaging of the mouse was performed using an IVIS Lumina XRMS Series III Imaging System (PerkinElmer, America) with a XGI-8 system.

Preparation of $\mathrm{Cr}^{3+}$-doped, $\mathrm{Nd}^{3+}$-doped, and $\mathrm{Cr}^{3+} / \mathrm{Nd}^{3+}$-codoped $\mathrm{B}-\mathrm{ZGC}$ NPs. The nanoparticles were produced by a hydrothermal method. The influences of dopant concentration, molar ratio of metallic ions, and molar ratio of between $\mathrm{NaHCO}_{3}$ and metallic ions on the particle size and photoluminescence were shown in Table S1-S3. Typically, $3.6 \mathrm{mmol} \mathrm{Zn}\left(\mathrm{NO}_{3}\right)_{2} \cdot 6 \mathrm{H}_{2} \mathrm{O}, 6 \mathrm{mmol} \mathrm{Ga}\left(\mathrm{NO}_{3}\right)_{3} \cdot x \mathrm{H}_{2} \mathrm{O}$, and $(0.003,0.006$, $0.012,0.024,0.048,0.096) \mathrm{mmol} \mathrm{Cr}\left(\mathrm{NO}_{3}\right)_{3} \cdot 9 \mathrm{H}_{2} \mathrm{O}$ were dissolved in $18 \mathrm{~mL}$ of Milli Q water. Then, $50.4 \mathrm{mmol}$ sodium hydrogen carbonate was quickly added to the above mixed solution with vigorous stirring. The suspension was subjected to ultrasound for $15 \mathrm{~min}$ and vigorously stirred for another $15 \mathrm{~min}$. The white suspension was then transferred into a Teflonlined autoclave $(30 \mathrm{~mL})$ and hydrothermally treated at $220{ }^{\circ} \mathrm{C}$ for $10 \mathrm{~h}$. The white precipitate was collected through centrifugation (Beckman Allegra 64R, USA) and washed with $0.01 \mathrm{M} \mathrm{HCl}$ acid solution until a transparent sediment was formed. The purified nanoparticles were dried in air at $45^{\circ} \mathrm{C}$.

In vitro cytotoxicity assay and cell labeling. The cytotoxicity of the modified NPs were tested using 4T1 cells and 4T1-luc cells by tetrazolium dye MTT assay. To further correlate the luminescence intensity of the A-ZGCN NPs and the labeled cells, 4T1-luc cells were cultured with A-ZGCN NPs for $24 \mathrm{~h}$. Then, A-ZGCN labeled 4T1-luc cells $\left(1 \times 10^{6}, 5 \times 10^{5}, 1 \times 10^{5}, 5 \times 10^{4}, 1 \times 10^{4}, 5 \times 10^{3}\right)$ in $100 \mu$ L RPMI medium were dispersed in black 96-well plates. The persistent luminescence images of A-ZGCN labeled 4T1-luc cells were collected after excitation by a $657 \mathrm{~nm}$ light-emitting diode 
(LED) lamp for $3 \mathrm{~min}$. The bioluminescence images of the A-ZGCN labeled 4T1-luc cells were collected after addition of $10 \mu \mathrm{L}(15 \mathrm{mg} / \mathrm{mL})$ D-luciferin firefly.

The A-ZGCN labeled 4T1-luc cells $\left(1 \times 10^{5}\right)$ with different generations $\left(1^{\text {st }}, 2^{\text {nd }}, 3^{\text {rd }}\right.$, $\left.4^{\text {th }}, 5^{\text {th }}\right)$ were cultured in RPMI medium for $24 \mathrm{~h}$ and collected by digestion. The afterglow luminescence was recorded on the CCD camera of an IVIS Lumina XRMS Series III Imaging System after excitation with a $657 \mathrm{~nm}$ LED lamp for $3 \mathrm{~min}$.

Animal model. Specific pathogen free (SPF) grade nude female mice (18-20 g) were purchased from Shanghai SLAC Laboratory Animal Co. Ltd. and used under protocols approved by the Laboratory Animal Center of Soochow University. The tumors were grafted by inoculation of $2 \times 10^{6} 4 \mathrm{~T} 1-\mathrm{luc}$ cells or A-ZGCN NPs labeled 4T1-luc cells in about $50 \mathrm{~mL}$ PBS into the breast fat pad of each female Balb/c mouse $(n=4)$.

Histopathology examination. The tumors and major organs (heart, liver, spleen, lung, kidney and axillary lymph nodes) were harvested from mice in each group after 18 days' therapy, and soaked in $4 \%$ polyformaldehyde solution. The tissue samples were sectioned into slices and stained with hematoxylin and eosin (H\&E). The images of the tumors and organ slices were observed under a digital microscope.

\section{References}

1. Liu, H.; Ren, F.; Zhang, H.; Han, Y.; Qin, H.; Zeng, J.; Wang, Y.; Sun, Q.; Li, Z.; Gao, M., Oral administration of highly bright $\mathrm{Cr}^{3+}$ doped $\mathrm{ZnGa}_{2} \mathrm{O}_{4}$ nanocrystals for in vivo targeted imaging of orthotopic breast cancer. J. Mater. Chem. B 2018, 6, 1508-1518. 
Table S1. Synthesis of different $\mathrm{Cr}^{3+}$-doped B-ZGC NPs.

\begin{tabular}{ccc}
$\mathrm{n}\left(\mathrm{Cr}^{3+}\right) /\left[\mathrm{n}\left(\mathrm{Zn}^{2+}\right)+\mathrm{n}\left(\mathrm{Ga}^{3+}\right)^{+}\right]$ & Size $/ \mathrm{nm}$ & Quantum yield $(\mathrm{QY}) / \%$ \\
$0.03125 \%$ & $12.8 \pm 1.8$ & 23.10 \\
$0.0625 \%$ & $14.3 \pm 2.7$ & 24.84 \\
$0.125 \%$ & $12.5 \pm 1.9$ & 25.51 \\
$0.25 \%$ & $10.5 \pm 1.8$ & 21.50 \\
$0.5 \%$ & $9.6 \pm 1.6$ & 12.19 \\
$1 \%$ & $11.7 \pm 1.8$ & 4.60 \\
\hline
\end{tabular}

Table S2. Different addition amounts of $\mathrm{NaHCO}_{3}$ during the preparation of nanoparticles

\begin{tabular}{ccc}
\hline $\mathrm{n}\left(\mathrm{NaHCO}_{3}\right) /\left[2 \mathrm{n}\left(\mathrm{Zn}^{2+}\right)+3 \mathrm{n}\left(\mathrm{Ga}^{3+}\right)\right]$ & Size $/ \mathrm{nm}$ & $\mathrm{QY} / \%$ \\
\hline $0.5: 1$ & $8.0 \pm 1.4$ & 3.61 \\
$1: 1$ & $7.5 \pm 0.9$ & 19.13 \\
$1.5: 1$ & $7.7 \pm 0.8$ & 24.55 \\
$2: 1$ & $12.5 \pm 1.9$ & 27.14 \\
$2.5: 1$ & -- & 25.51 \\
$3: 1$ & -- & 26.30 \\
\hline
\end{tabular}


Table S3. Synthesis of different Cr/Nd-doped B-ZGCN NPs

\begin{tabular}{cc}
\hline $\mathrm{n}\left(\mathrm{Cr}^{3+}\right) / \mathrm{n}\left(\mathrm{Nd}^{3+}\right)$ & $\mathrm{QY} / \%$ \\
$0: 1$ & 12.09 \\
$1: 1$ & 15.83 \\
$1: 10$ & 19.20 \\
$1: 20$ & 19.47 \\
$1: 40$ & 21.26 \\
$1: 80$ & 21.54 \\
$1: 100$ & 24.14 \\
\hline
\end{tabular}



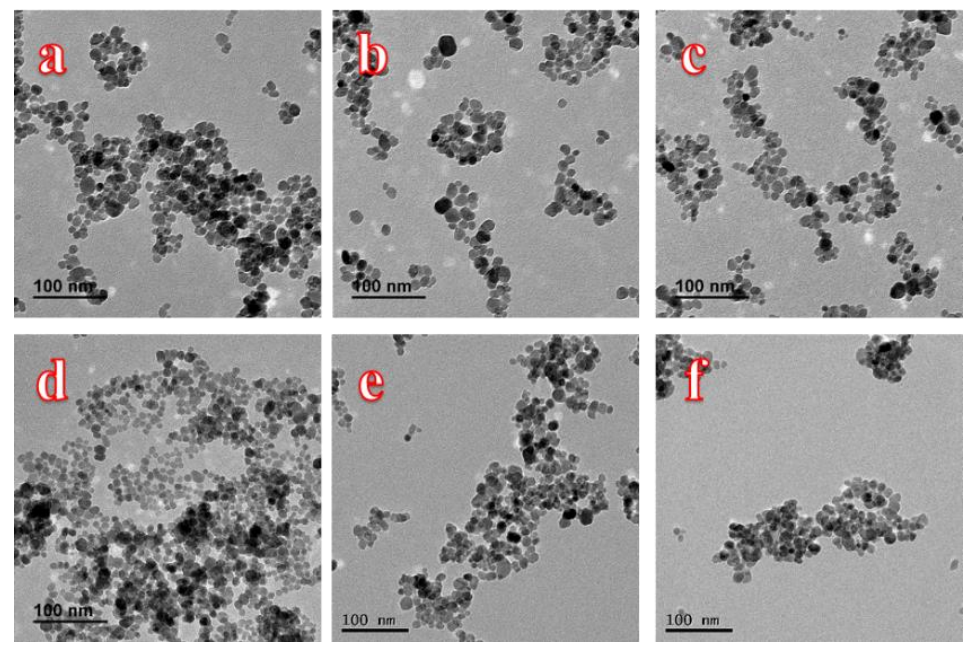

Figure S1. TEM images of B-ZGC nanoparticles doped with different contents of $\mathrm{Cr}^{3+}$ ions: (a) $0.03125 \%$, (b) $0.0625 \%$, (c) $0.125 \%$, (d) $0.25 \%$, (e) $0.5 \%$, (f) $1 \%$.
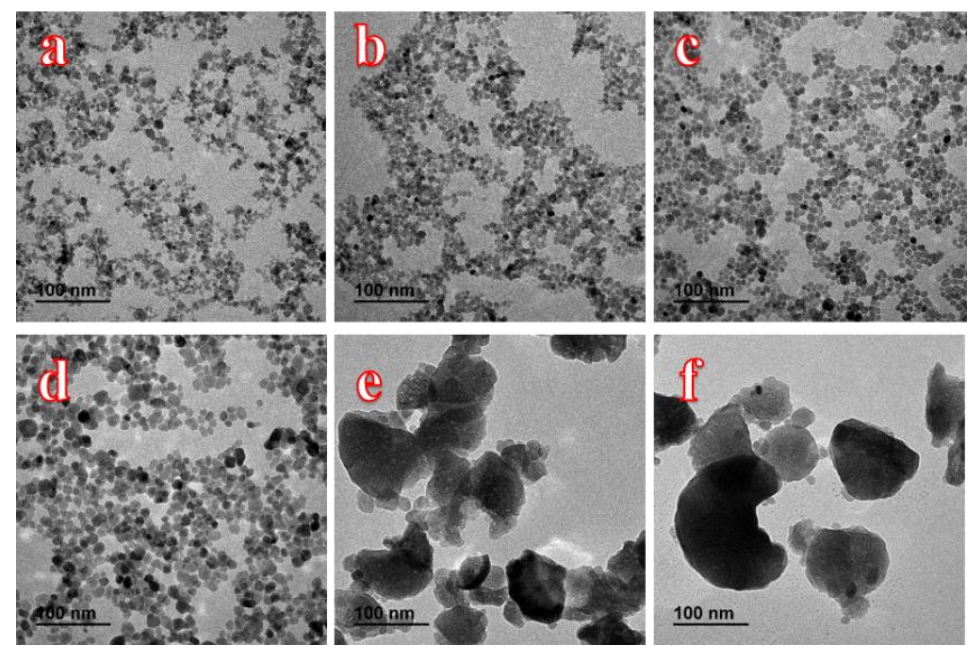

Figure S2. TEM images of B-ZGCN nanoparticles prepared with different ratios of

$\mathrm{NaHCO}_{3} n\left(\mathrm{NaHCO}_{3}\right) /\left[2 \mathrm{n}\left(\mathrm{Zn}^{2+}\right)+3 \mathrm{n}\left(\mathrm{Ga}^{3+}\right)\right]:$ (a) $0.5: 1$, (b) 1:1, (c) 1.5:1, (d) 2:1, (e) 2.5:1, (f) $3: 1$. 

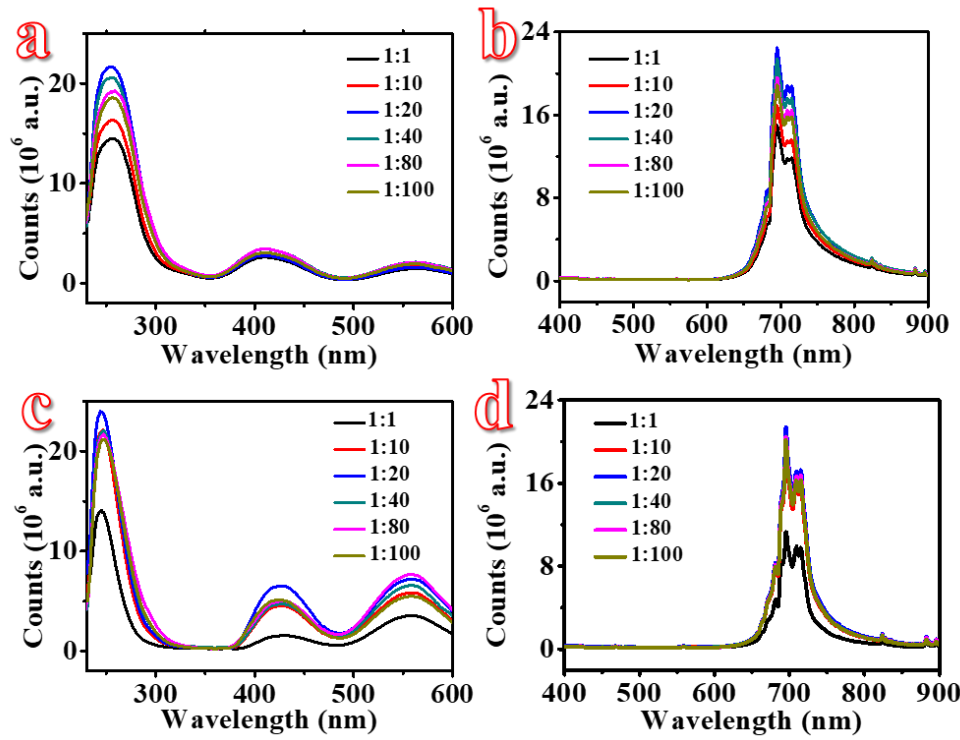

Figure S3. The optical properties of ZGCN NPs doped with various ratios of $\mathrm{Cr}^{3+} / \mathrm{Nd}^{3+}$ ions. (a) Excitation (emission at $696 \mathrm{~nm}$ ), (b) Photoluminescence emission spectra (excitation at $254 \mathrm{~nm}$ ) of B-ZGCN NPs before annealing treatment. (c) Excitation (emission at $696 \mathrm{~nm}$ ), (d) Photoluminescence emission spectra (excitation at $254 \mathrm{~nm}$ ) of A-ZGCN NPs after annealing treatment. 

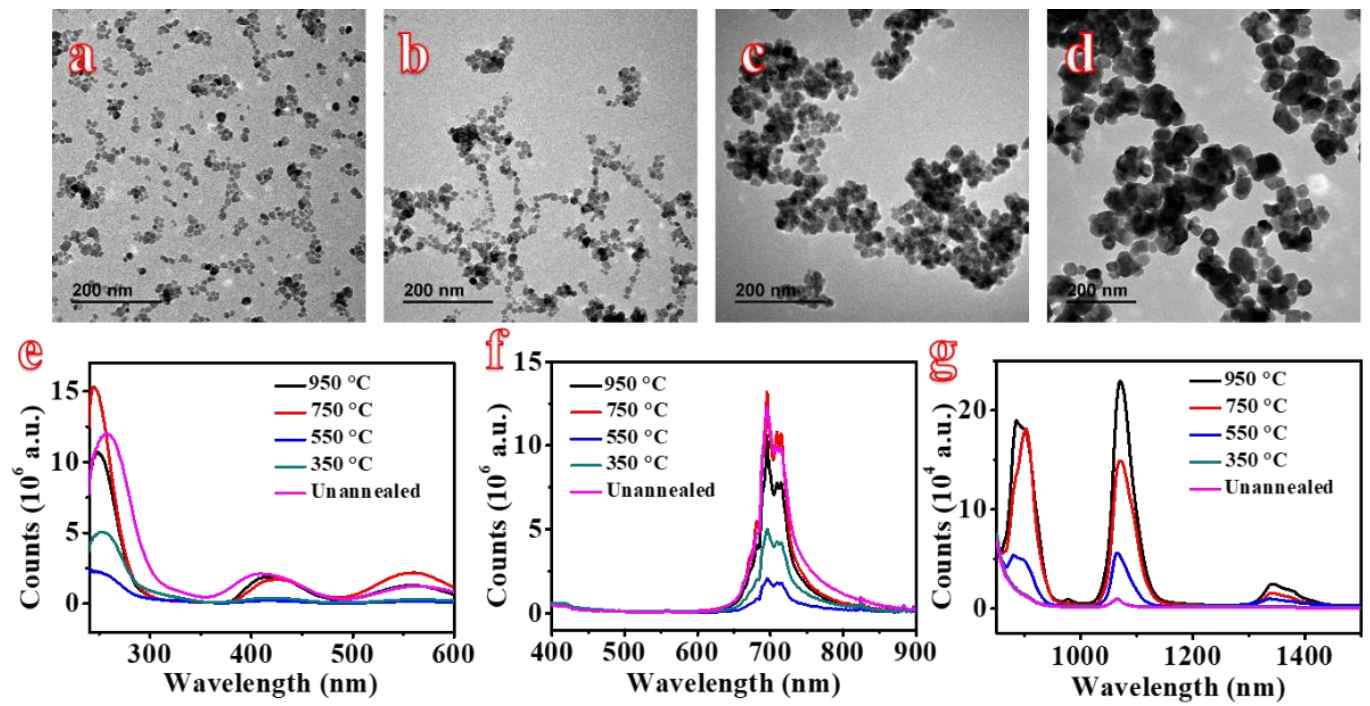

Figure S4. TEM images of ZGCN NPs after annealing at with (a) $350{ }^{\circ} \mathrm{C}$, (b) $550{ }^{\circ} \mathrm{C}$,

(c) $750{ }^{\circ} \mathrm{C}$, and (d) $950{ }^{\circ} \mathrm{C}$, respectively. (e) Excitation spectra (emission at $696 \mathrm{~nm}$ ), (f)

NIR-I (excitation at $254 \mathrm{~nm}$ ) and (g) NIR-II photoluminescence spectra (excitation at $808 \mathrm{~nm}$ ) of ZGCN NPs after annealing at different temperatures.
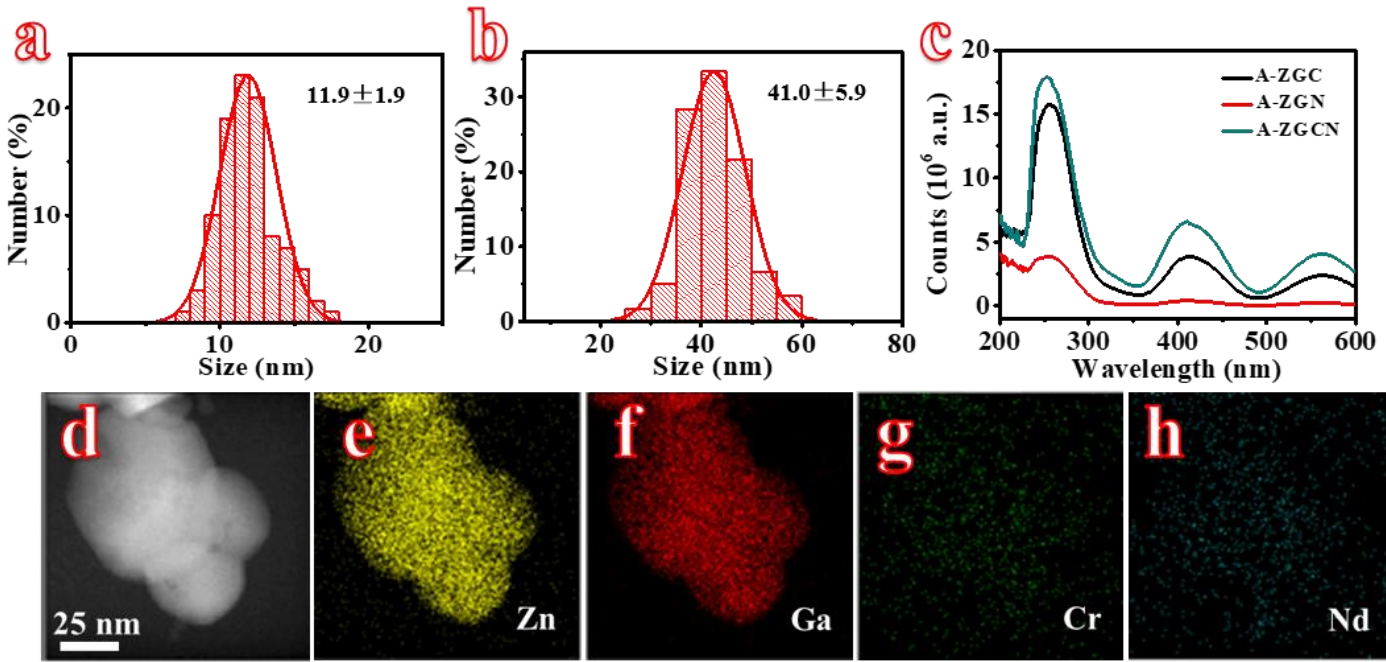

Figure S5. Size distributions of (a) B-ZGCN NPs and (b) A-ZGCN NPs; (c) Excitation spectra of A-ZGC, A-ZGN, and A-ZGCN nanopowders under irradiation of $254 \mathrm{~nm}$ xenon lamp; (d) HAADF-STEM images and (e-h) EDX elemental mapping of AZGCN NPs. 

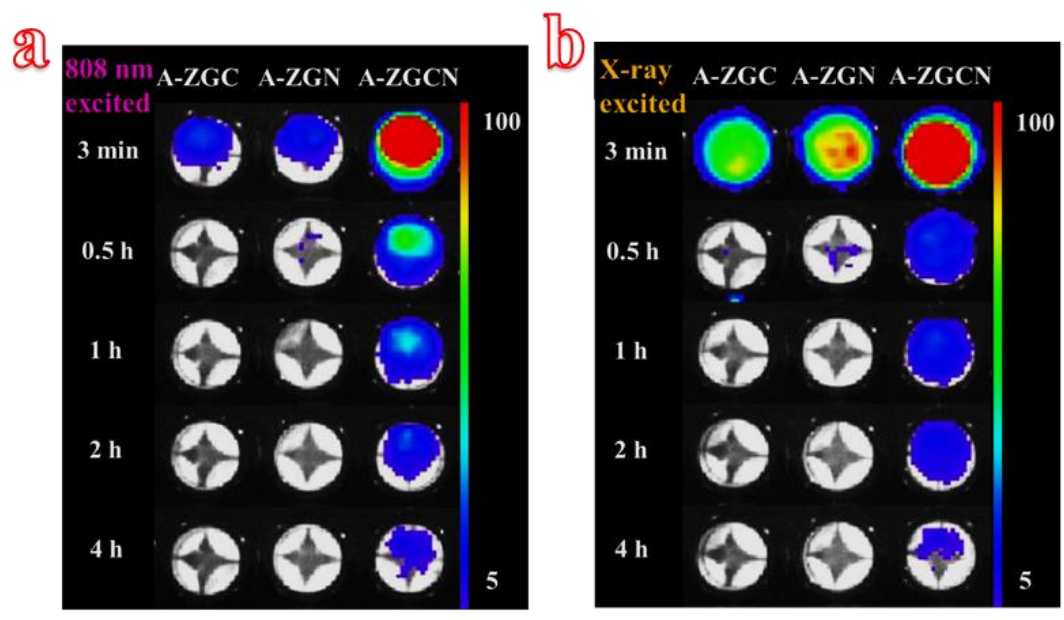

Figure S6. In vitro NIR persistent luminescence imaging of A-ZGC, A-ZGN, and AZGCN NPs after irradiation by (a) an $808 \mathrm{~nm}$ laser $\left(100 \mathrm{~mW} / \mathrm{cm}^{2}, 5 \mathrm{~min}\right)$ and (b) Xrays $(25 \mathrm{kV}, 1 \mathrm{~min})$.
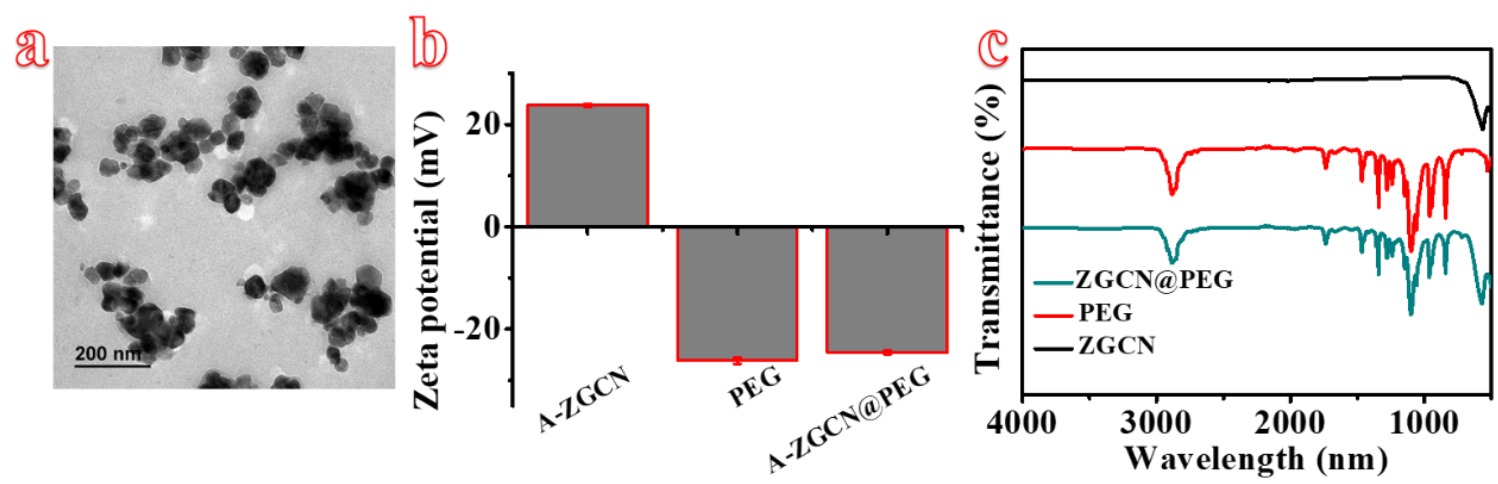

Figure S7. (a) TEM image of A-ZGCN@PEG NPs, (b) zeta potential, and (c) FTIR spectra of these A-ZGCN NPs after surface modification. 

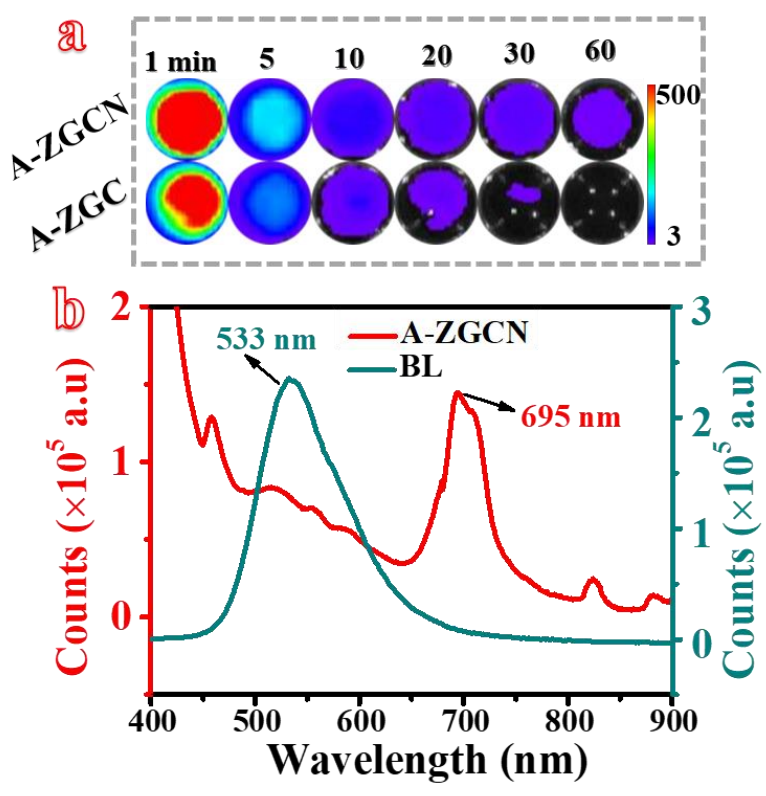

Figure S8. (a) The near-infrared persistent luminescence collected at different time points $(1,5,10,20,30,60 \mathrm{~min})$ for $2 \times 10^{6} \mathrm{~A}-\mathrm{ZGCN}$ and $2 \times 10^{6} \mathrm{~A}-\mathrm{ZGC}$ NPs labeled 4T1-luc cells after irradiation by a $254 \mathrm{~nm}$ lamp. (b) The photoluminescence and bioluminescence spectra of suspensions of 4T1-luc cells labeled with A-ZGCN NPs (excited at $365 \mathrm{~nm}$ ).
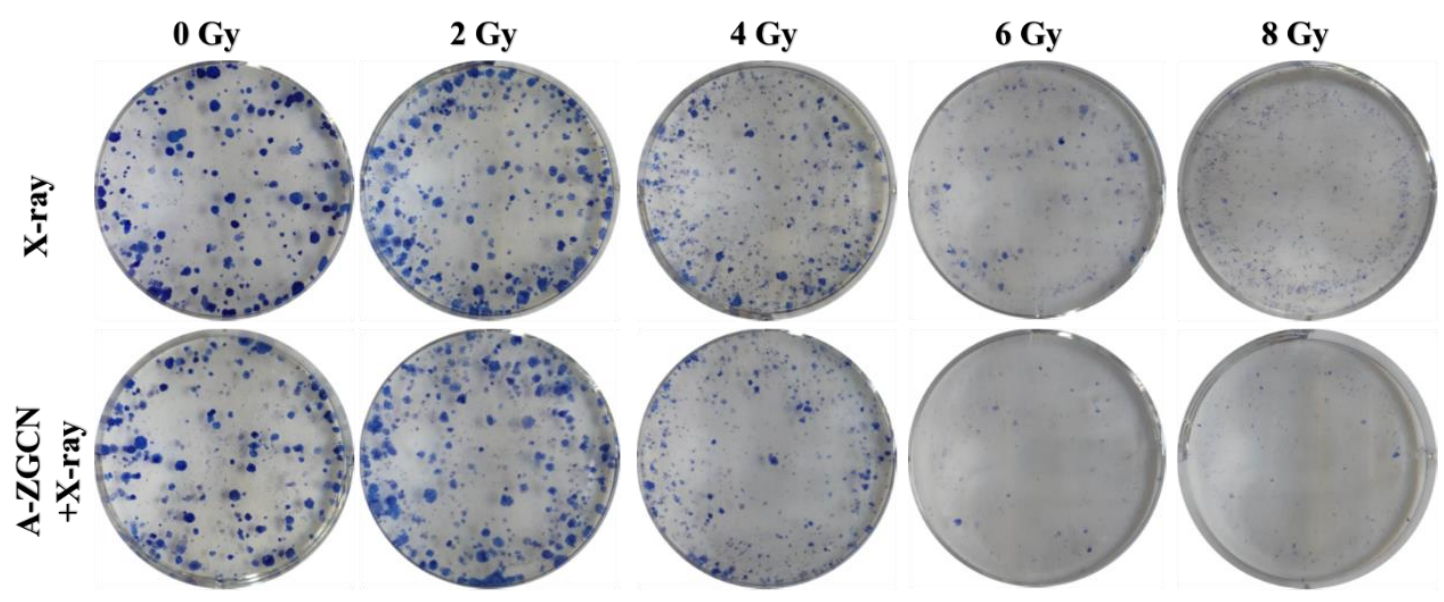

Figure S9. Photograph from colony formation assay for 4T1 cells in the presence and absence of A-ZGCN NPs. 

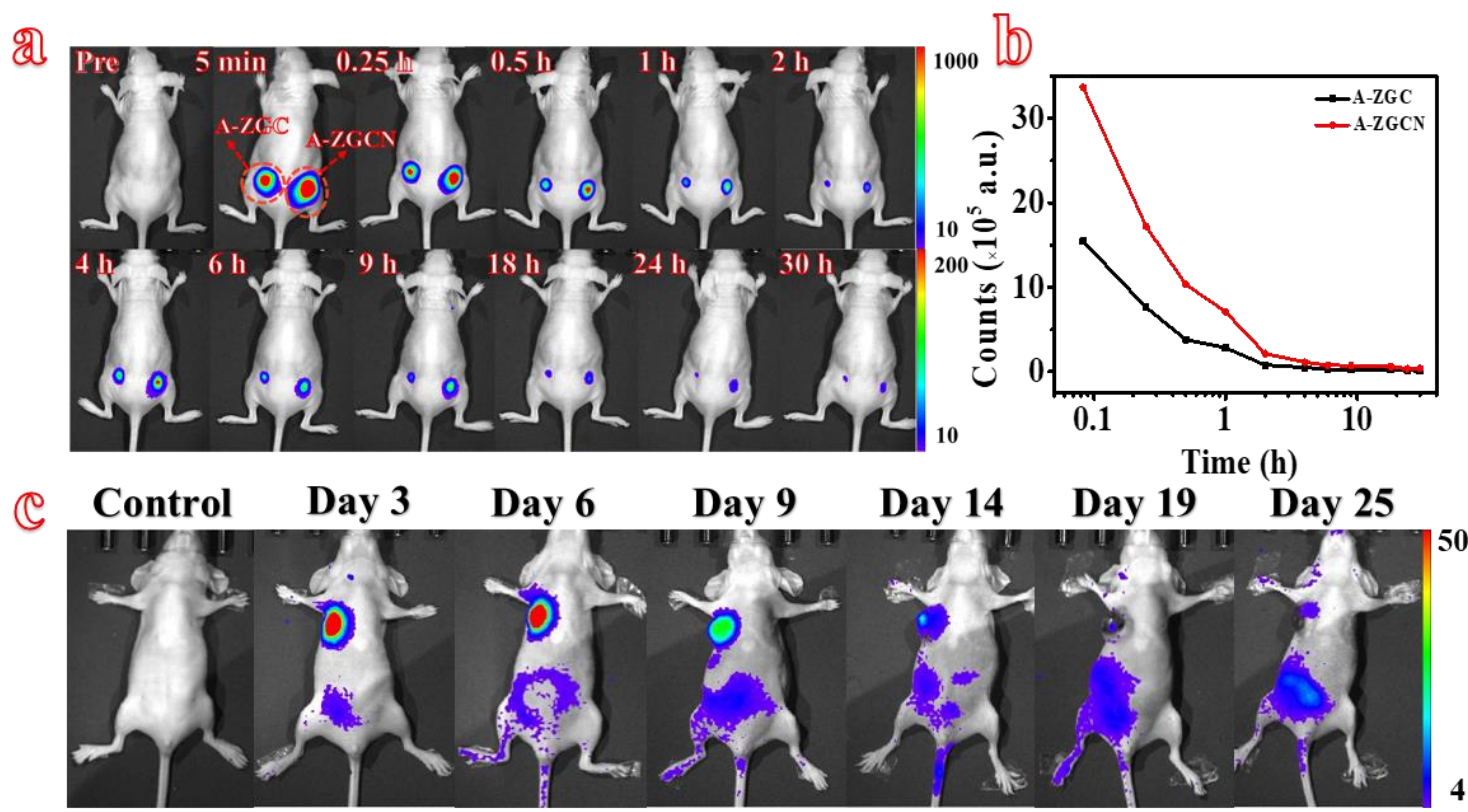

Figure S10. In vivo NIR luminescent images (a) and corresponding quantitative luminescence attenuation (b) of a normal mouse subcutaneously injected with AZGC@PEG and A-ZGCN@PEG NPs ( 5 mg/mL, $50 \mu \mathrm{L})$, after excitation by $254 \mathrm{~nm}$ UV light for 5 min. (c) In vivo afterglow luminescence images of a tumor-bearing mouse at different time points after the transplantation of A-ZGCN-labeled 4T1-luc cells. 


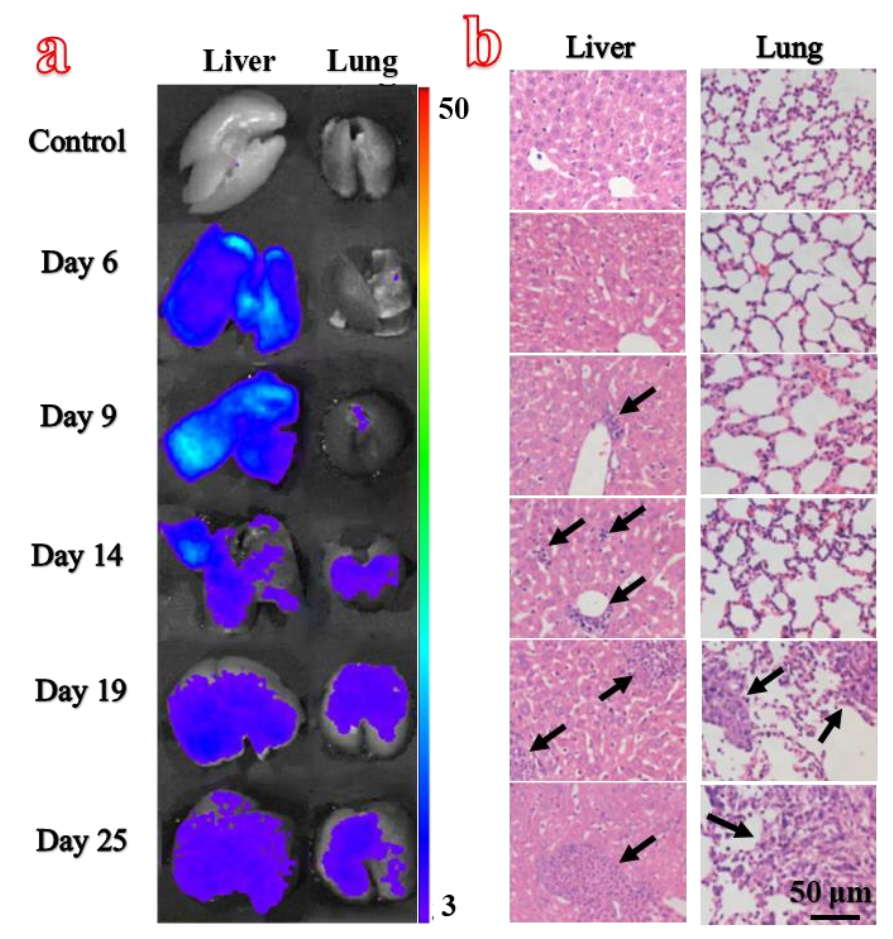

Figure S11. (a) Persistent luminescence images and (b) H\&E staining of liver and lung collected after different numbers of days. 


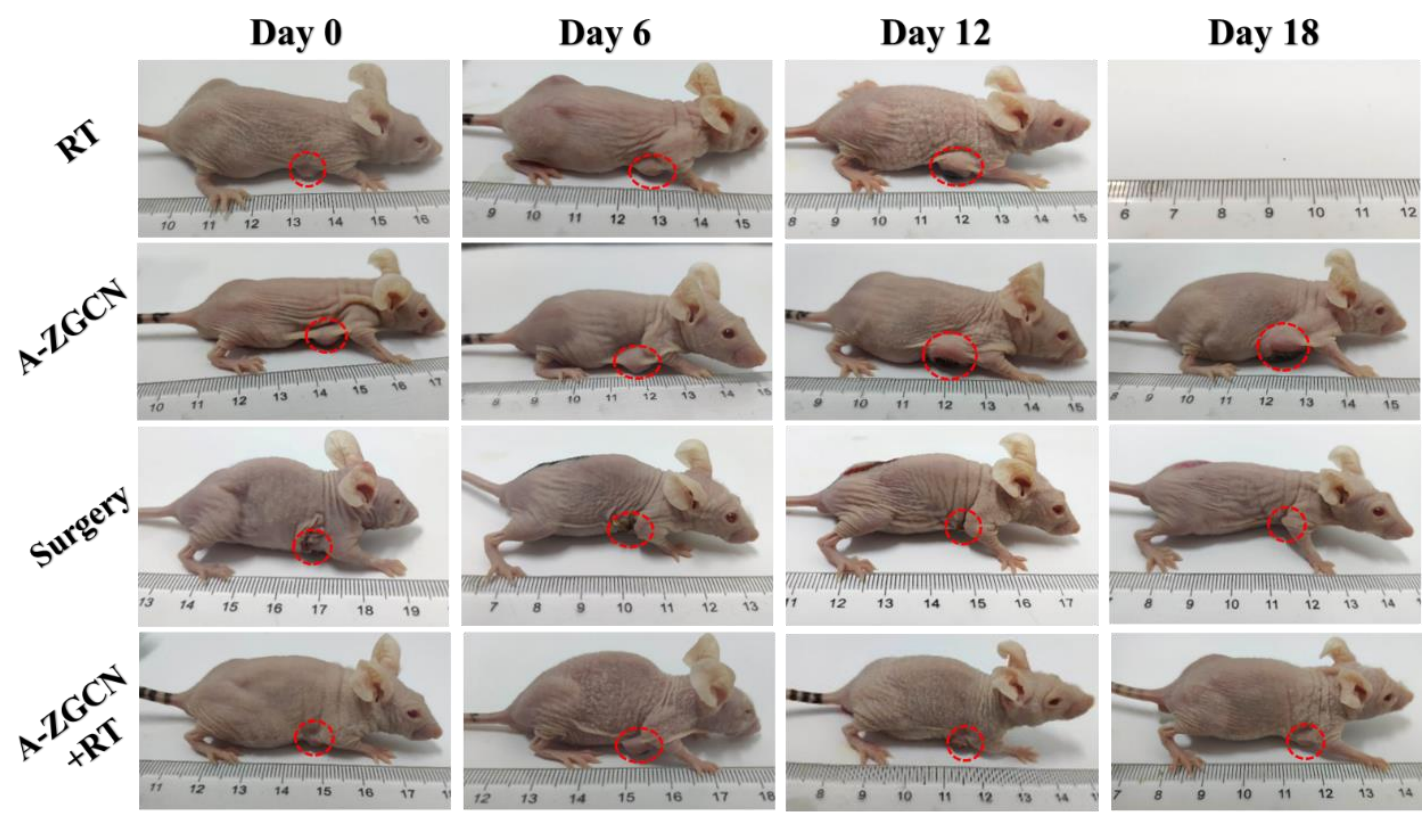

Figure S12. Representative photographs of different groups of mice recorded on various days after treatments. 